\title{
A 67-Year-Old Woman With Laryngeal Tuberculosis
}

\author{
Ali Amiri ${ }^{1, *} ;$ Vahid Almasi $^{2}$ \\ ${ }^{1}$ Department of Internal Medicine, Lorestan University of Medical Sciences, Khorramabad, IR Iran \\ ${ }^{2}$ Clinical Research Center, Lorestan University of Medical Sciences, Khorramabad, IR Iran \\ *Corresponding author: Ali Amiri, Department of Internal Medicine, Lorestan University of Medical Sciences, Khorramabad, IR Iran. E-mail: ali_pul_amiri@yahoo.com
}

Received: February 15, 2014; Accepted: April 12, 2014

\begin{abstract}
A 67-year-old woman presented with dysphagia, odynophagia and hoarseness. In her chest X-ray, there was evidence of consolidation associated with loss of volume in the upper lobe of the left lung. Acid-fast bacillus was not seen at the direct smear of the throat exudates. In laryngoscopy, bilateral edema of the arytenoids and exophytic lesions at the posterior commissure were detected. In the biopsy sample of these lesions, acid-fast bacilli were seen. Finally, the patient was treated by anti-tuberculosis drugs.
\end{abstract}

Keywords: Hoarseness; Laryngeal tuberculosis; Mycobacterium tuberculosis

\section{Introduction}

Laryngeal tuberculosis is a granulomatous disease of the larynx. It is an uncommon condition and it occurs in $1-10 \%$ of all cases of tuberculosis [1]. Laryngeal tuberculosis may cause odynophagia, dysphagia and hoarseness. Laryngeal tuberculosis had non-pathognomonic complaints and physical findings, thus the diagnosis of laryngeal tuberculosis may be difficult.

\section{Case Presentation}

A 67-year-old woman came to the clinic of Lorestan University of Medical Sciences in Khorramabad with dysphagia and odynophagia. Dysphagia and odynophagia had started from 2 months before. These symptoms had been progressive and the patient could not drink and eat anything at the arrival time. In addition, she had had cough and hemoptesis from 2 months before. She had had fever, shiver and weigh loss of 10 - $15 \mathrm{~kg}$ per 2 months. She had had hoarseness from 20 days before the arrival. She was a passive smoker, but she did not use cigarettes, alcohol and narcotics. In physical examinations, her oral cavity was found to be dry. There were thick, dense and yellow exudates on the erythematous mucosa of the throat. Her white blood cells were 10700 (per/ $\mu \mathrm{L}$ ), and $79 \%$ and 29\% of them were neutrophil and lymphocyte, respectively. Her erythrocyte sedimentation rate was $46 \mathrm{~mm} / \mathrm{h}$. Her C reactive protein was 1.160 . The amount of the red blood cells, platelets, hemoglobin, hematocrit, serum electrolytes, thyroid and liver function tests were in the normal range. In her chest X-ray, there was evidence of consolidation (acinar shadow) associated with loss of volume in the upper lobe of the left lung, resulting in narrowing and deviation of the trachea to the left side and mildly elevation of the left hemi-diaphragm.

Acid-fast bacillus was not seen at the direct smear of the throat exudates. In laryngoscopy, bilateral edema of the arytenoids and exophytic lesions at the posterior commissure and above the glottis were detected. A biopsy sample was afforded from these lesions. In the biopsy sample, multinucleated giant cell were seen in Hematoxylin and Eosin staining (Figure 1).

Based on the chest X-ray findings and the pathologic evaluation of the biopsy sample, diagnosis of tuberculosis was made. The patient was treated by isoniazid (300 mg/day), rifampin (300 mg/BD), pyrazinamid (1 g/day) and ethambutol (500 mg/day). After 2 weeks, the general condition of the patient improved significantly, she could drink and eat and the exudates of the throat were removed.

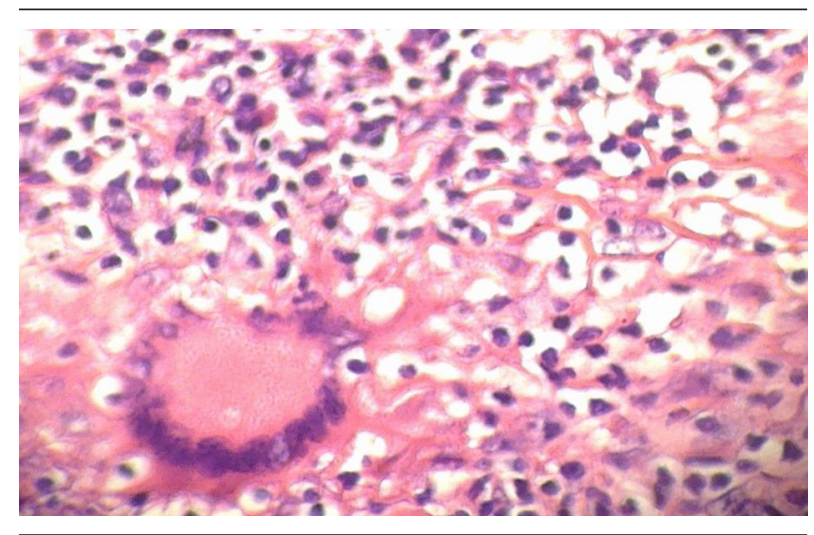

Figure 1. A Multinucleated Giant Cell Were Seen in Hematoxylin and Eosin Staining 


\section{Discussion}

Tuberculosis is a granulomatous infectious disease. It is caused by Mycobacterium tuberculosis. It is a life threatening infection and a major cause of disability and death. Tuberculosis primarily affects the lungs. However, 10-15\% of all tuberculosis cases have extra pulmonary tuberculosis [2]. Tuberculosis of the larynx is the most frequent laryngeal granulomatous disease. Laryngeal tuberculosis can be a primary condition, or a secondary condition to pulmonary tuberculosis [3]. Harney et al. reported that laryngeal tuberculosis had been observed in 15-37\% of the pulmonary tuberculosis patients [4]. However, Krecicki et al. reported that $1.5 \%$ of the patients with active pulmonary tuberculosis had laryngeal tuberculosis. Laryngeal tuberculosis can cause dysphagia, odynophagia and hoarseness [5]. Other reported symptoms of laryngeal tuberculosis are dyspnea, stridor, cough and hemoptysis [6]. Systemic symptoms are rare [7]. Wang et al. reported that the most frequent symptom of the laryngeal tuberculosis was hoarseness [8], while Topak et al. reported that odynophagia was the most common complaint among the patients with laryngeal tuberculosis [9].

Our case was a 67-year-old woman. She had the general symptoms of the tuberculosis such as cough, hemoptesia, fever, shiver and weigh loss. Furthermore, there was evidence of consolidation (acinar shadow) associated with loss of volume in the upper lobe of the left lung in her chest X-ray. She told that she had had dysphagia and odynophagia from 2 months and hoarseness from 20 days before the arrival. In conclusion, we recommend that laryngeal tuberculosis be considered as a differential diagnosis, especially in the patients with pulmonary tuberculosis.

\section{Acknowledgements}

The authors are grateful to the Clinical Research Center of Lorestan University of Medical Sciences, Dr Hadi Majidi (Radiologist) and Mr Yadollah Pournia (MS, Instructor of English Language).

\section{Funding/Support}

Lorestan University of Medical Sciences.

\section{References}

1. Hermani B, Sawitra D. Laryngeal tuberculosis: an important issue. Acta Med Indones. 2006;38(1):29-32.

2. Memon GA, Khushk IA. Primary tuberculosis of tongue.J Coll Physicians Surg Pak. 2003;13(10):604-5.

3. Kruschinski C, Welkoborsky HJ. Tuberculosis of the larynx associated with orofacial granulomatosis in childhood. Otolaryngol Head Neck Surg. 2005;132(6):967-9.

4. Harney M, Hone S, Timon C, Donnelly M. Laryngeal tuberculosis: an important diagnosis. J Laryngol Otol. 2000;114(11):878-80.

5. Krecicki T, Zalesska-Krecicka M, Zatonski T, Jankowska R, Skrzydlewska-Kaczmarek B. Laryngeal tuberculosis. Lancet Infect Dis. 2004;4(1):57.

6. Smulders YE, De Bondt BJ, Lacko M, Hodge JA, Kross KW. Laryngeal tuberculosis presenting as a supraglottic carcinoma: a case report and review of the literature. J Med Case Rep. 2009;3:9288.

7. Lim JY, Kim KM, Choi EC, Kim YH, Kim HS, Choi HS. Current clinical propensity of laryngeal tuberculosis: review of 60 cases. Eur Arch Otorhinolaryngol. 2006;263(9):838-42.

8. Wang CC, Lin CC, Wang CP, Liu SA, Jiang RS. Laryngeal tuberculosis: a review of 26 cases. Otolaryngol Head Neck Surg. 2007;137(4):582-8.

9. Topak M, Oysu C, Yelken K, Sahin-Yilmaz A, Kulekci M. Laryngeal involvement in patients with active pulmonary tuberculosis. Eur Arch Otorhinolaryngol. 2008;265(3):327-30. 